\title{
BMJ Open Global Influenza Hospital-based Surveillance Network (GIHSN): results of surveillance of influenza and other respiratory viruses in hospitalised patients in Brazil, 2015
}

Sonia M Raboni, ${ }^{1,2}$ Fernanda EA Moura, ${ }^{3}$ Braulia C Caetano, ${ }^{4}$ Valéria M Avanzi, ${ }^{5}$ Luciane A Pereira, ${ }^{2}$ Meri B Nogueira, ${ }^{2}$ Luine R Vidal, ${ }^{2}$ Isabel CF Tavares, ${ }^{6,7}$ Florence K Pradel, ${ }^{8}$ Valentina S Picot, ${ }^{8}$ Joan Puig-Barbera, ${ }^{9}$ Marilda M Siqueira ${ }^{4}$

To cite: Raboni SM, Moura FEA, Caetano BC, et al. Global Influenza Hospital-based Surveillance Network (GIHSN): results of surveillance of influenza and other respiratory viruses in hospitalised patients in Brazil, 2015. BMJ Open 2018;8:e017603. doi:10.1136/ bmjopen-2017-017603

- Prepublication history for this paper is available online. To view these files, please visit the journal online (http://dx.doi. org/10.1136/bmjopen-2017017603).

Received 10 May 2017 Revised 22 September 2017 Accepted 8 December 2017

Check for updates

For numbered affiliations see end of article.

Correspondence to Dr Sonia M Raboni; sraboni@ufpr.br

\section{ABSTRACT}

Background Influenza-like illness occurs annually worldwide, with peak timing and severity varying seasonally, resulting in significant annual mortality. Objectives There were three objectives: (1) to describe the epidemiological and clinical features of hospitalised patients with severe acute respiratory infection caused by influenza and other respiratory viruses (ORVs); (2) to report the influenza seasonality in the region and (3) to correlate findings of influenza circulation and immunisation time in Brazil.

Patients/methods This study took place in three Brazilian hospitals located in cities with different climatic conditions (Curitiba (south), Rio de Janeiro (south-east) and Fortaleza (north-east)). Patients presenting with an acute process with indication for admission consisting of a predefined set of conditions potentially associated with recent influenza infection were enrolled.

Results We screened 1666 patients, with 595 meeting the inclusion criteria. Influenza viruses and ORVs were detected in $6.5 \%$ and $59 \%$ of patients, respectively. Influenza-positive cases fell into the severe spectrum as compared with those with ORVs (30\% vs $11 \%$ ), but without any difference in mortality rates. Epidemiological results revealed variations in the peak time of influenza infections between north-east (Fortaleza) and south (Curitiba) Brazil, basically following the rain period of each region. In north-east Brazil, viral circulation was prevalent in the first 4 months of the year, indicating that the vaccination campaign occurred in a postseasonal period, possibly explaining the low effectiveness.

Conclusions The active-surveillance model is a valuable tool for investigating respiratory virus impact on hospitalised patients, with influenza-infection monitoring enabling implementation of adequate preventive measures.

\section{INTRODUCTION}

Influenza-like illness (ILI) occurs annually worldwide, but peak timing and severity of the epidemic vary seasonally. ${ }^{1}$ Although
Strengths and limitations of this study

- This study used the same model of active surveillance applied in other outside centres allowing subsequent comparisons on the impact of influenza and other respiratory viruses in hospitalised patients worldwide.

- More information was added on seasonality and severity of the influenza disease in South America, whose data are limited.

- Our data reinforce the growing consensus that vaccinations in Northeast Brazil are being carried out in a post-seasonal period, with consequent low effectiveness.

- The low inclusion of patients in Rio de Janeiro that does not allow for an assessment of the impact of viral respiratory infections in southeastern Brazil.

- The study was carried out with data collected in 1 year, which limits predictions on the impact of influenza longitudinally in these locales.

surveillance for antigenic drift or influenza-virus shift is intense, annual mortality still exceeds 250000 and results usually from complications, such as pneumonia, neurological events and circulatory failure. ${ }^{2}$

Seasonal influenza epidemics peak during the winter in temperate latitudes of both the northern and southern hemispheres. By contrast, tropical countries might experience two annual peaks, with shorter and less intense epidemics. ${ }^{34}$ Moreover, previous studies showed that influenza activity is frequently out of phase with the hemispherical winter in many tropical regions and some temperate ones, with the consequence that optimal timing for routine influenza-vaccination recommendations does not necessarily correspond to the activity expected for that hemisphere. ${ }^{5}$ 
Brazil, which includes temperate and tropical regions, exhibits an important regional heterogeneity in influenza peak. ${ }^{67}$ Seasonal influenza epidemics are usually associated with 'cold-dry' and 'humid-rainy' climates; ${ }^{8}$ however, in addition to climate and environmental conditions, host factors, such as age, pre-existing immunity, genetic polymorphisms and presence of comorbidities, are associated with seasonal variations of this infection, vaccine responsiveness, as well as the severity of influenza epidemics. ${ }^{9}$

Vaccination is the main public health measure used to reduce the frequency of severe influenza cases. ${ }^{2}$ In Brazil, influenza vaccination has been performed annually since 1999. Initially, immunisation targeted individuals aged $\geq 65$ years; however, in 2000 , the age limit was lowered to 60 years. In 2016, the Brazilian Ministry of Health extended the recommendation to children $<5$ years, pregnant women, people with chronic non-communicable diseases and health professionals. ${ }^{10-12}$ Overall, in 2015, national immunisation campaigns achieved a vaccination coverage of $82.7 \%$ in priority groups. ${ }^{13}$

The Global Influenza Hospital-based Surveillance Network (GIHSN) is a public-private partnership between research institutes, hospitals and several laboratories around the world, and was established to study the epidemiology of severe influenza in consecutive seasons in different countries. (http://www.gihsn.org/?page= map). In 2015, this network comprised a total of 31 collaborating sites from seven countries, including Brazil. All participants follow the same core investigation protocol developed by the GIHSN scientific committee. Through broad geographical coverage and data standardisation, the GIHSN group seeks to obtain a global picture of the impact of influenza on patients with respiratory pathologies. Additionally, this network provides invaluable tools to investigate the seasonality of influenza in regions where this information is not available, as well as a framework for estimating the effectiveness of seasonal influenza vaccines in preventing severe cases among age and risk groups.

In 2015, the GIHSN group began a study in Rio de Janeiro, Fortaleza and Curitiba, three cities located in distinct regions of Brazil and enabling the evaluation of effects of different climatic conditions on the seasonality of influenza infections. Here, we report the initial findings of this surveillance project, including seasonal activity and the clinical and epidemiological features observed.

\section{PATIENTS AND METHODS}

All procedures were performed according to approved protocols, and consent was obtained from all patients (or guardians).

\section{Study design and site description}

This multicentre, cross-sectional study was designed to provide information on active surveillance of influenza in hospitalised patients. Data were collected in 2015 from three participating hospitals located in distinct cities and regions of Brazil: Curitiba (south), Fortaleza (north-east) and Rio de Janeiro (south-east) (figure 1).

In Curitiba and Rio de Janeiro, patient recruitment and data collection occurred from April to September, coinciding with the period of influenza seasonality in south and south-east Brazil. In Fortaleza, the study spanned all of 2015, because, despite a previous study showing influenza circulation between January and April ${ }^{14}$ the seasonality of influenza in this region is not well established.

In Curitiba, the study was performed at Hospital de Clínicas da Universidade Federal do Paraná (HC/UFPR), a 310-bed, tertiary care, academic centre, with 47 beds in the intensive care unit (ICU). It is also among the sentinel hospitals for influenza and severe acute respiratory infection (SARI) surveillance in the region. Curitiba is located at $25^{\circ} 25^{\prime} \mathrm{S}, 49^{\circ} 15^{\prime} \mathrm{W}, 924 \mathrm{~m}(3031 \mathrm{ft})$ and has a mild marine west coast climate, with no dry season and warm summers. Seasonality is moderate, with heavy precipitation during mild winters and a mean temperature of $16.5^{\circ} \mathrm{C}$. The city population numbered $\sim 1879355$ people in 2015 .

In Fortaleza, this study was conducted at Hospital Infantil Albert Sabin, a public health institution of 270 beds. Fortaleza is a city of 2500000 inhabitants and is located at sea level, $4^{\circ}$ south of the equator. Its tropical climate is characterised by two distinct seasons: a rainy one occurring between January and May, and a dry one occurring during the rest of the year. There is high relative humidity $(79 \%)$ and little variation in the average temperature $\left(26.4^{\circ} \mathrm{C}\right)$.

In Rio de Janeiro, this study was conducted at Hospital Quinta D'Or, which is a private hospital with 350 beds for general admission and 150 beds in the ICU. Rio de Janeiro is located at $22^{\circ} 54^{\prime} \mathrm{S}, 43^{\circ} 12^{\prime} \mathrm{W}$, and its climate is classified as tropical Atlantic. The year comprises two seasons: a hot and relatively humid one and another with mild temperatures and less rainfall.

\section{Patient recruitment and inclusion/exclusion criteria}

Patients of all ages who were admitted for any acute condition described as potentially associated with influenza were considered eligible for enrolment in the study. To be included, eligible patients were identified in the hospital admission registries and had to comply with all of the following criteria: (1) admission within 24 hours to 72 hours prior to recruitment; (2) a resident of the area of the study (defined for each site) and belonging to the base population source; and (3) a referral history for any acute condition associated with influenza and exhibiting onset within $\leq 7$ days prior to hospital admission. This study used the European Centre for Disease Control (CDC) definition for ILI, which included at least one of four systemic symptoms (fever, headache, myalgia or malaise) and at least one of three respiratory symptoms (cough, sore throat or shortness of breath). Patients who did not give consent to participate, institutionalised individuals and those with a history of hospitalisation $\leq 30$ days prior to recruitment were excluded from the study. 


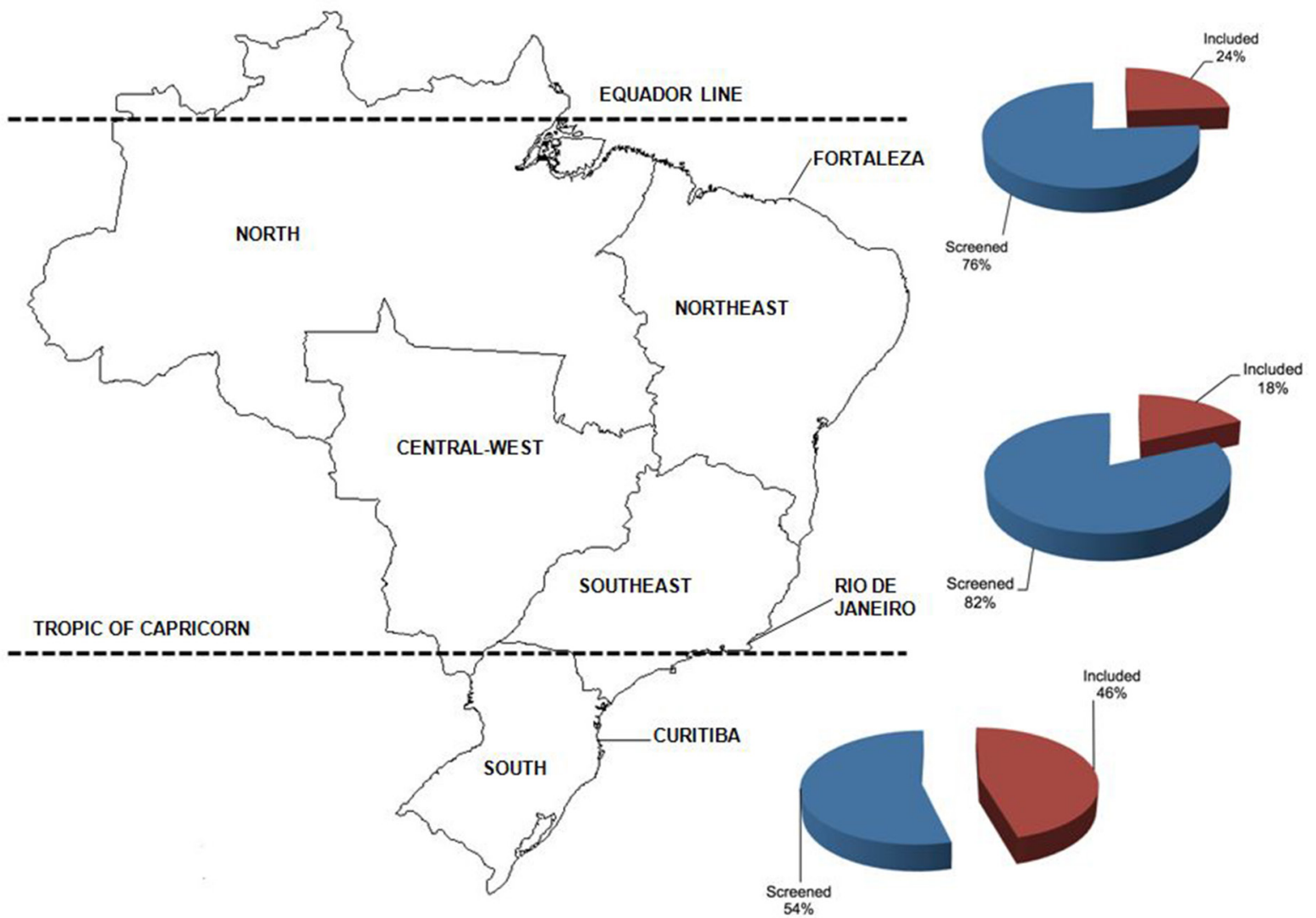

Figure 1 Map of Brazil showing the location of Curitiba, Rio de Janeiro and Fortaleza and the total of screened (blue) and included (red) patients at each site.

\section{Sample collection and processing}

Samples were collected using nylon FLOQswabs (Copan, Brescia, Italy) and included one nasopharyngeal and one pharyngeal swab (or nasal swab for patients $<14$ years). Samples were added to a flask containing $3 \mathrm{~mL}$ of universal viral-transport medium (Copan) and stored at $-80^{\circ} \mathrm{C}$ until testing.

\section{Sample testing to detect influenza and other respiratory viruses (ORVs)}

All sampleswere submitted for nucleic acid extraction using a QIAmp viral RNA mini kit (Qiagen, Hilden, Germany). The presence of influenza A (FLUA) (H1N1pdm and H3N2) and influenza B (FLUB) (B/Yamagata or B/ Victoria) was analysed by reverse transcription real-time PCR (RT-PCR) according to CDC protocol. ${ }^{15} 16$

In a subset of samples $(n=497)$, the presence of other pathogens was assessed using a commercial multiplex RT-PCR assay (FTD respiratory pathogens 21 kit; FastTrack Diagnostics, Esch-sur-Alzette, Luxembourg) designed to detect the following: FLUA (H1N1pdm09); FLUB; rhinovirus (RHV); coronavirus (COV) genotypes NL63, 229E, OC43 and HKU1; parainfluenza (PIV) types 1, 2, 3 and 4; human metapneumovirus A/B (HMPV); bocavirus (BOV); respiratory syncytial virus A/B (RSV); adenovirus (ADV); enterovirus (EV); parechovirus; and the atypical bacterium Mycoplasma pneumonia.
Alternatively, we used the Seeplex RV15 ACE detection kit (Seegene, Seoul, Korea), a multiplex PCR-based assay allowing for the simultaneous detection of the following viruses: ADV; HMPV; PIV types 1, 2, 3 and 4; FLUA; FLUB; RSV types A and B; RHV types A, B and C; EV; BOV; and COV types 229E/NL63 and OC43/HKU1. Both tests were performed according to manufacturer's protocol.

\section{Data analysis}

Data were compiled using JMP software V.5.2.1 (SAS Institute, Cary, North Carolina, USA) and analysed using GraphPad Prism V.5.03 (GraphPad Software, La Jolla, California, USA). Baseline demographic and clinical characteristics with normal and non-normal distributions were presented as the mean $\pm \mathrm{SD}$ and medians with IQRs, respectively. Epidemiological and clinical data comparing patients with influenza infection and those with ORV infection were assessed; however, only monoinfected cases were included in this analysis. Fisher's exact test, $\chi^{2}$ test or the Wilcoxon-Mann-Whitney test was performed as appropriate. A multivariate logistic regression model was fitted to evaluate clinical or epidemiological characteristics of virus-positive cases to determine association with disease severity. Variables included age, presence of comorbidities and fatal outcome. All statistical tests were two-sided and considered significant at $\mathrm{P}<0.05$. 


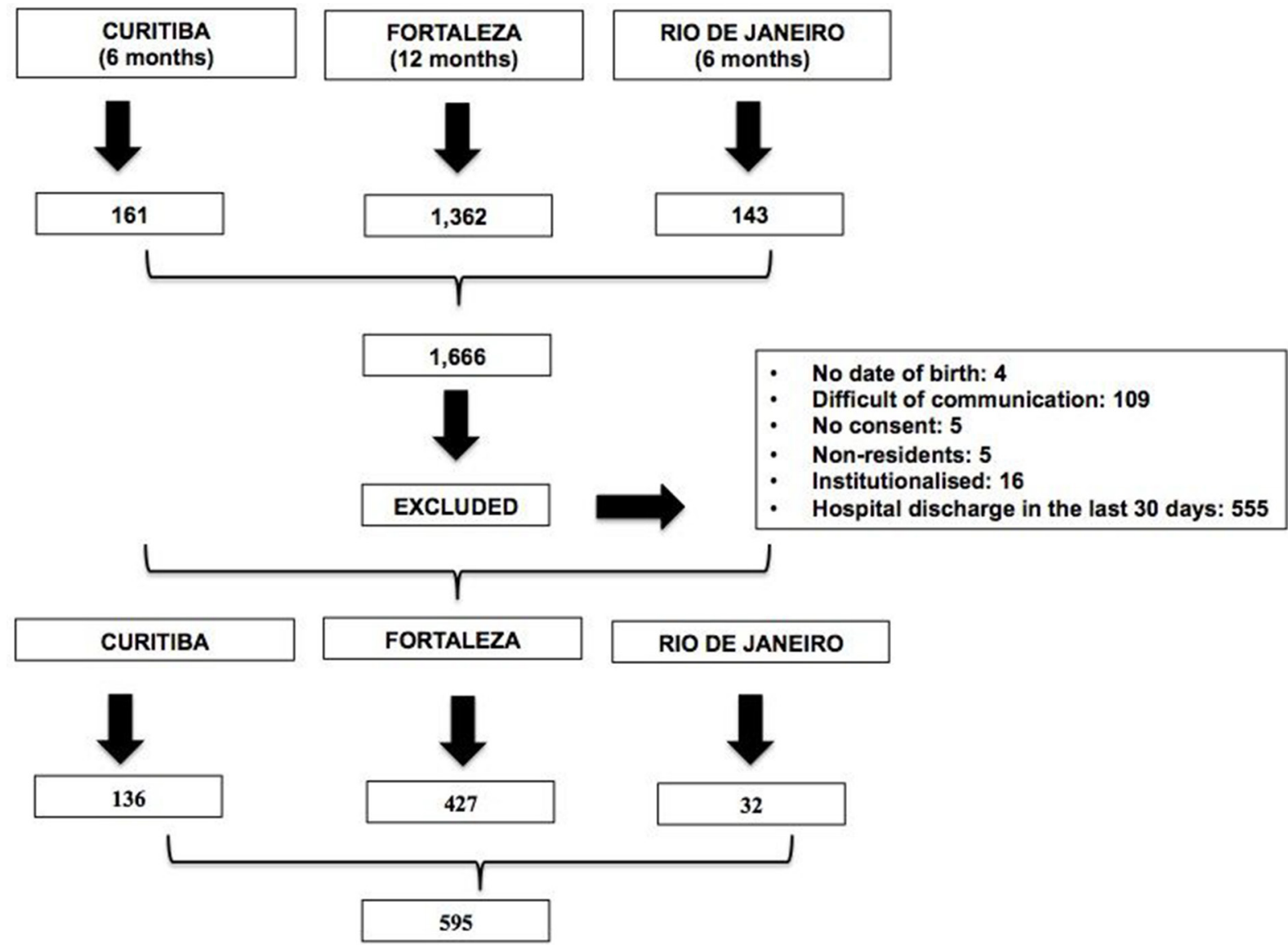

Figure 2 Flow chart summarising the process of patient inclusion in the study performed in GIHSN hospitals in Curitiba, Fortaleza and Rio de Janeiro over 6 months, 12 months and 6 months of collection, respectively. GIHSN, Global Influenza Hospital-based Surveillance Network.

We defined severe disease as that requiring mechanical ventilation, admission to an ICU or resulting in a fatal outcome. To improve our understanding of the seasonality of influenza infections at the study sites, we evaluated the monthly distribution of samples and the viruses detected by RT-PCR. This information was then plotted against the temperature and precipitation (historical means) recorded for each month.

\section{RESULTS}

Demographics and epidemiological characteristics of the study population

During the study period, 1666 patients from three participating hospitals were screened (161 in Curitiba, 1362 in Fortaleza and 143 in Rio de Janeiro), with 595 patients meeting the inclusion criteria (figure 2).

The demographic and clinical characteristics of the population are listed in table 1 . In the Curitiba and Fortaleza subsets, men and women were recruited in equal proportions, with most individuals $<5$ years of age. By contrast, in Rio de Janeiro, women and adults were predominant, as the hospital did not evaluate children.

As expected from a predominantly young population, the prevalence of chronic conditions associated with aggravation of influenza was low in the Curitiba and Fortaleza subsets, whereas the Rio de Janeiro subset was characterised by a significantly higher prevalence of conditions, such as cardiovascular disease, chronic obstructive pulmonary disease and diabetes.

Other factors that impact the prevalence of severe influenza infections are the use of antivirals and vaccination. Antiviral administration was more frequently reported in Curitiba. Overall, influenza vaccination rates were low, despite most age groups included in the study being covered by the Brazilian immunisation programme: $\sim 31 \%$ of patients from Curitiba were vaccinated, and an estimated $14 \%$ and $19 \%$ were vaccinated in Fortaleza and Rio de Janeiro, respectively. Only 21\% of children $<5$ years who were included in the study were vaccinated. Severe disease was detected in 149/595 cases (24\%), but mortality was low: $9 / 595$ (1.5\%).

\section{Influenza and ORV evaluation}

All samples were tested for influenza virus, with 39 (6.5\%) positive: 23 (59\%) FLUA H3N2 and $16(41 \%)$ for FLUB (all Yamagata-like lineages). Of these cases, nine (23\%) presented ORV co-infection.

The presence of ORV was tested in 497 patients (83.5\%), with $293(59 \%)$ positive and $46(16 \%)$ presenting co-infection. RSV was the most frequently identified virus and the main source of co-infections $(n=27 ; 49 \%)$, whereas 
Table 1 Epidemiological and clinical characteristics of the patients included in this study

\section{Characteristics}

Sex

Male

Female

Age (median, years/IQR)

Age group (years)

$\begin{array}{ll}<2 & 86 \\ 2-4 & 23 \\ 5-17 & 11 \\ 18-64 & 12 \\ >65 & \end{array}$

Time to onset of symptoms (median, days/IQR)

Exposure to smoking

\begin{tabular}{|c|c|c|c|}
\hline Yes & $22(16)$ & $109(25)$ & $1(3)$ \\
\hline No & $90(66)$ & 277 (65) & $21(66)$ \\
\hline Ex-smoker & $24(18)$ & $41(10)$ & $10(31)$ \\
\hline \multicolumn{4}{|l|}{ Chronic diseases* } \\
\hline None & $76(56)$ & $341(78)$ & $9(28)$ \\
\hline Cardiovascular & $16(12)$ & $26(6)$ & $12(38)$ \\
\hline Pulmonary & $32(23)$ & $8(2)$ & $7(22)$ \\
\hline Asthma & $13(10)$ & 37 (9) & $9(28)$ \\
\hline Diabetes & $1(1)$ & $3(1)$ & $6(19)$ \\
\hline Immunosuppressed & $2(1.5)$ & $9(2)$ & $1(3)$ \\
\hline Renal & 0 & $7(1.5)$ & $2(6)$ \\
\hline Rheumatic disease & 0 & $8(2)$ & 0 \\
\hline Cirrhosis & 0 & $2(0.5)$ & $2(6)$ \\
\hline Neuromuscular & $11(8)$ & $22(5)$ & 0 \\
\hline Neoplasm & $1(1)$ & $1(0.2)$ & $5(16)$ \\
\hline Autoimmune & $1(1)$ & $8(2)$ & $1(3)$ \\
\hline Length of stay in hospital (median days/IQR) & $2(1-53)$ & $1(0-14)$ & $2(1-53)$ \\
\hline \multicolumn{4}{|l|}{ Polypnoea } \\
\hline Yes & $108(80)$ & $51(12)$ & $17(53)$ \\
\hline No & 7 (5) & $303(71)$ & $3(9)$ \\
\hline $\mathrm{NI}$ & $21(15)$ & $73(17)$ & $12(38)$ \\
\hline \multicolumn{4}{|l|}{ Use of antiviral drugs } \\
\hline Yes & $13(10)$ & $10(2)$ & 0 \\
\hline Days of use (median/IQR) & $5(2-5)$ & $15(1-35)$ & - \\
\hline \multicolumn{4}{|l|}{ Vaccination in 2014} \\
\hline Yes & $42(31)$ & $59(14)$ & $6(19)$ \\
\hline No & $94(69)$ & $344(81)$ & $26(81)$ \\
\hline $\mathrm{NI}$ & 0 & $24(5)$ & 0 \\
\hline \multicolumn{4}{|l|}{ ICU admission } \\
\hline Yes & $37(27)$ & $10(2)$ & $26(81)$ \\
\hline No & $99(73)$ & $417(98)$ & $6(19)$ \\
\hline \multicolumn{4}{|l|}{ Mechanical ventilation } \\
\hline Yes & 29 (21.3) & $13(3.0)$ & 0 \\
\hline
\end{tabular}

Continued

\section{Curitiba ( $n=136)(\%) \quad$ Fortaleza $(n=427)(\%) \quad R J(n=32)(\%)$}

$\begin{array}{lll}61(45) & 243(57) & 12(37) \\ 75(55) & 184(43) & 20(63) \\ 1.4(0.5-87.9) & 1.3(0-16.6) & 1.4(3.3-89.3)\end{array}$

$86(63)$

$23(17)$

11 (8)

$12(9)$

$4(3)$

254 (59)

0

$100(24)$

3 (9)

$73(17) \quad 4(12)$

0

9 (28)

$16(51)$

$2(0-7)$

0

$2(0-6)$

$2(0-7)$

?.

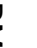




\begin{tabular}{lllc}
\hline Table 1 Continued & & & \\
\hline Characteristics & Curitiba (n=136) (\%) & Fortaleza $(\mathbf{n}=\mathbf{4 2 7})(\mathbf{\%})$ & $\mathbf{R J}(\mathbf{n}=\mathbf{3 2})(\mathbf{\%})$ \\
\hline No & $107(78.7)$ & $414(97.0)$ & 0 \\
Death & & & \\
Yes & $4(3)$ & $4(1)$ & $1(3)$ \\
No & $132(97)$ & $423(99)$ & $31(97)$ \\
Severe disease & $99(73)$ & $16(4)$ & $26(81)$ \\
\hline
\end{tabular}

*Some patients presented more than one comorbidity.

ICU, intensive care unit; NI, no information; RJ, Rio de Janeiro.

influenza co-infections were found in only $23 \%$ (9/39) of cases. The respiratory pathogens detected in this subset of samples are reported in figure 3 .

\section{Severe cases and mortality associated with influenza and ORV} As shown in table 2, 9/37 influenza-positive cases fell into the severe spectrum, and severe disease was significantly more frequent in patients with influenza infection than in those with ORV infection ( $30 \%$ vs $11 \%$ ). In an adjusted analysis, only age was associated with this outcome $(\mathrm{P}=0.01)$, whereas the presence of comorbidities was not $(\mathrm{P}=0.12)$. No difference in mortality was observed between patients with influenza infection and patients with ORV infection

\section{Seasonality of influenza infections in two geographical regions of Brazil}

Comparison of the seasonality of influenza infections with temperature and precipitation (historical means) recorded for each month revealed variations between the north-east (Fortaleza) and south (Curitiba) regions of Brazil in relation to the peak period of influenza infections; however, in both regions, influenza coincided with the peak of the rainy season. Our results indicated that immunisation against influenza in the north-east was performed after the period of virus circulation (figure 4), whereas data from Rio de Janeiro were not evaluated due to the small number of patients included in the study.

\section{DISCUSSION}

GIHSN was established with the main goal of improving the understanding of roles of influenza infections in the development of severe respiratory diseases. The information gathered by this network can potentially contribute to the elucidation of influenza seasonality in some regions, implementation of more efficient containment measures and the improvement of preventive interventions such as immunisation.

In Brazil, the Ministry of Health employs two systems for epidemiological assessment of respiratory diseases: sentinel surveillance of ILI and universal surveillance of SARI. Sentinel surveillance consists of a network of designated healthcare units (public or private) distributed throughout the country, in which random samples of respiratory cases are periodically collected for detection and genetic characterisation of circulating viruses. In universal SARI surveillance, all severe respiratory cases admitted to ICUs and all deaths related to respiratory disease are investigated in the laboratory. ${ }^{17}$ In the present study, only hospitalised patients identified by an active

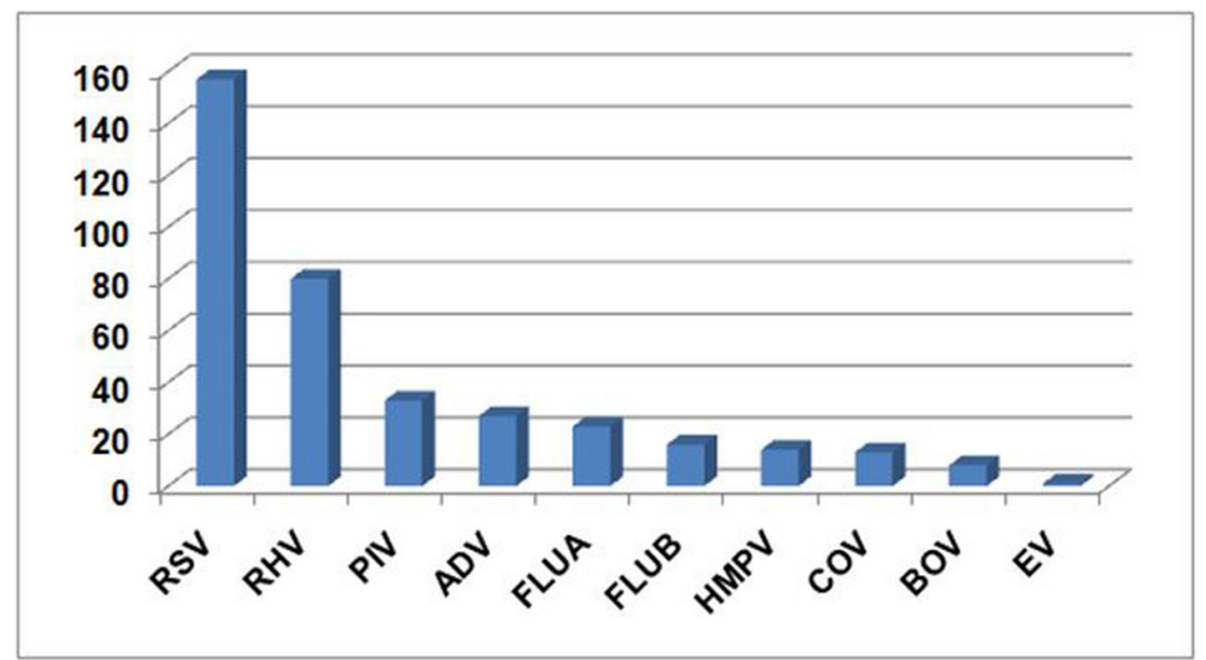

Figure 3 Respiratory viruses detected in a subset of samples ( $n=497)$ from patients included in the study. ADV, adenovirus; BOV, bocavirus; COV, coronavirus; EV, entrovirus; FLUA, influenza A; FLUB, influenza B; HMPV, human metapneumovirus; RHV, rhinovirus; RSV, respiratory syncytial virus; PIV, parainfluenza. 
Table 2 Epidemiological and clinical characteristics of patients with positive diagnosis for respiratory viruses treated in reference hospitals in Curitiba, Fortaleza and Rio de Janeiro, 2015

\begin{tabular}{|c|c|c|c|}
\hline \multirow[b]{2}{*}{ Characteristics } & \multicolumn{3}{|l|}{ Positive cases } \\
\hline & $\begin{array}{l}\text { Influenza virus } \\
\mathrm{n}=30(\%)\end{array}$ & $\begin{array}{l}\text { ORV } \\
n=247(\%)\end{array}$ & $\mathbf{P}$ \\
\hline \multicolumn{4}{|l|}{ Sex } \\
\hline Male & $13(43)$ & $121(49)$ & 0.5694 \\
\hline Female & $17(57)$ & $126(51)$ & - \\
\hline Age (median, years/IQR) & $2.6(1.3-5.9)$ & $0.8(0.2-2.0)$ & $<0.0001$ \\
\hline \multicolumn{4}{|l|}{ Age group (years) } \\
\hline$<2$ & $9(37)$ & $180(73)$ & 0.0002 \\
\hline $2-4$ & $10(33)$ & $43(17)$ & - \\
\hline $5-17$ & $4(13)$ & $15(6)$ & - \\
\hline$>18$ & $5(17)$ & $9(4)$ & - \\
\hline Time to onset of symptoms (median, days/IQR) & $1.5(0-4)$ & $2(1-4)$ & 0.1882 \\
\hline \multicolumn{4}{|l|}{ Chronic diseases $^{\star}$} \\
\hline No & $16(53)$ & $192(78)$ & 0.0064 (OR $3.095 \% \mathrm{Cl} 1.4$ to 6.6$)$ \\
\hline Pulmonary & $6(20)$ & $40(16)$ & - \\
\hline Cardiovascular & $7(23)$ & $16(6)$ & - \\
\hline Other & $3(10)$ & $27(11)$ & - \\
\hline Length of stay in hospital (median days/IQR) & $2(1-6)$ & $1.5(1-9.7)$ & 0.8726 \\
\hline \multicolumn{4}{|l|}{ Polypnoea } \\
\hline Yes & $11(37)$ & $88(36)$ & 0.8325 \\
\hline No & $15(50)$ & $139(56)$ & - \\
\hline $\mathrm{NI}$ & $4(13)$ & $20(8)$ & - \\
\hline \multicolumn{4}{|l|}{ Use of antiviral drugs } \\
\hline Yes & $1(3)$ & $9(4)$ & NA \\
\hline Days of use (median/IQR) & 5 & $5(5-35)$ & - \\
\hline \multicolumn{4}{|l|}{ Vaccination in 2014} \\
\hline Yes & $4(13)$ & $28(11)$ & 0.7634 \\
\hline No & $26(87)$ & $216(87)$ & - \\
\hline $\mathrm{NI}$ & 0 & $3(2)$ & - \\
\hline \multicolumn{4}{|l|}{ ICU admission } \\
\hline Yes & $8(27)$ & $22(9)$ & $\mathbf{0 . 0 0 8 1}$ (OR $3.795 \% \mathrm{Cl} 1.4$ to 9.3 ) \\
\hline No & $22(73)$ & $225(91)$ & - \\
\hline \multicolumn{4}{|l|}{ Mechanical ventilation } \\
\hline Yes & $6(20)$ & $15(6)$ & $0.0164(\mathrm{OR} 3.8,95 \% \mathrm{Cl} 1.3$ to 10.9$)$ \\
\hline No & $24(80)$ & $232(94)$ & - \\
\hline \multicolumn{4}{|l|}{ Death } \\
\hline Yes & $1(3)$ & $6(2)$ & 0.5006 \\
\hline No & $29(97)$ & $241(98)$ & - \\
\hline \multicolumn{4}{|l|}{ Severe disease } \\
\hline Yes & $9(30)$ & $26(11)$ & $\mathbf{0 . 0 0 6 1}(\mathrm{OR} 3.6,95 \% \mathrm{Cl} 1.5$ to 8.7 ) \\
\hline No & $21(70)$ & $221(89)$ & - \\
\hline
\end{tabular}

Bold numerals indicate statistically significant values.

*Some patients presented more than one comorbidity.

ICU, intensive care unit; NA, not applicable; NI, no information; ORV, other respiratory virus. 


\section{Curitiba}

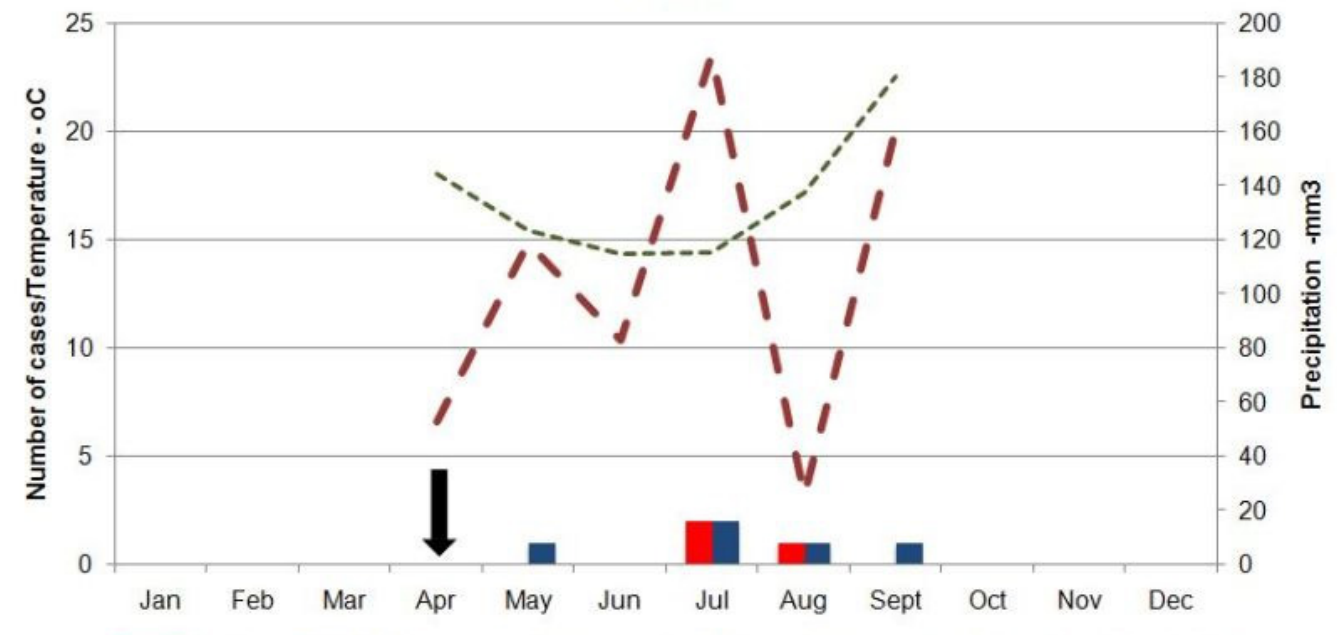

FLUA FLUB ---Average monthly temperature - - Average monthly rainfall

Fortaleza

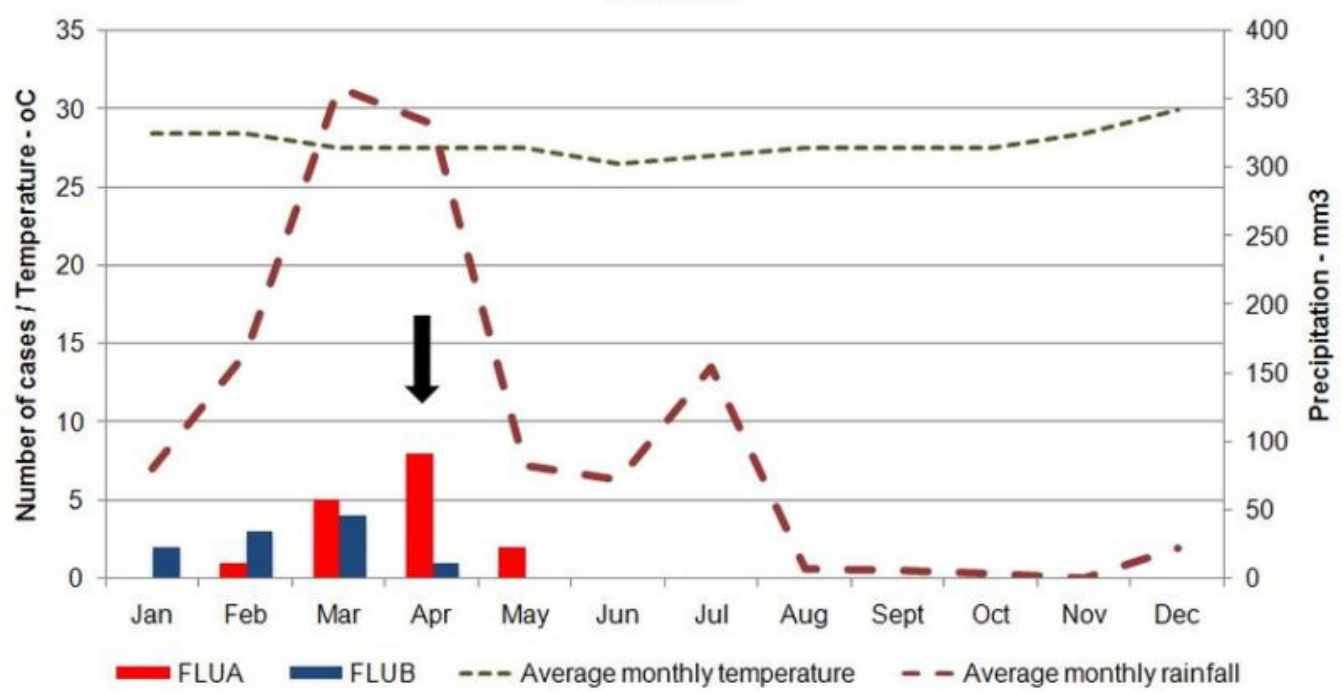

Figure 4 Number of detected cases of influenza A and B associated with meteorological data in Fortaleza (north-east) and Curitiba (south) in 2015. The time of the influenza-vaccination campaign is indicated by an arrow. Green and red lines represent mean temperatures and precipitation, respectively.

search of the participating health centres were included. In contrast to the Brazilian universal SARI surveillance protocol, which is restricted to ICUs, screening for our study included patients admitted to other wards and presenting diseases other than respiratory-related. Individuals admitted with conditions associated with aggravation of influenza infections, such as cardiovascular disease and diabetes, were also screened, thereby increasing the number of identified cases.

Overall, the frequency of influenza viruses in the study specimens was $6.5 \%$ (39/595), with FLUA comprising $60 \%$ of the flu-positive samples. This low prevalence reflected the profile of the 2015 influenza season in Brazil and in most of the Southern Hemisphere and tropical regions. During that year, viral activity remained low through the entire season, with higher prevalence of H3N2 and lower detection of FLUB and H1N1pdm09. Even in the temperate regions of the southern hemisphere, activity remained at an interseasonal level. In Brazil, a total of 11 945 cases of SARI were reported in 2015, of which 1089 $(9 \%)$ were related to influenza infection. Among these cases, 599 (55\%) were FLUA/H3N2, 234 (21.5\%) were FLUB, $141(12.9 \%)$ were FLUA/H1N1pdm09 and 115 $(10.5 \%)$ were reported as non-subtyped FLUA. ${ }^{18}$

Since 2014, the Brazilian Ministry of Health has included children $\leq 4$ years of age in the group of individuals at risk of severe influenza and subsequently began vaccinating this subpopulation. Data from the National Immunisation Programme show coverage rates $>83 \%$ among young children and pregnant women, $89 \%$ in the elderly and $95 \%$ in health workers. ${ }^{13}{ }^{17}$ In the present study, we observed that there was a higher percentage of patients from Curitiba vaccinated among the three sites; however, an overall lower coverage was observed, with only $21 \%$ of patients reporting or showing proof of influenza vaccination. This was surprising, considering that 
a significant number of our patients fell in at least one risk category or presented comorbidities. Given a $10.5 \%$ mortality rate due to influenza-related SARI cases in 2015, it is essential to assess the reasons for such low immunisation frequency. Among influenza deaths, $65 \%$ of patients and particularly those aged over 60 years presented at least one risk factor likely to cause complications. ${ }^{13}$ Our data emphasised the need for comprehensive identification of the weaknesses in the vaccination campaigns and an intensification of the efforts to bring immunisation to these individuals.

We also evaluated the prevalence of ORV in a subset of samples from HC/UFPR and in samples selected randomly from among patients from Fortaleza and Rio de Janeiro. Overall, different respiratory viruses were found in 59\% of the tested samples. This was consistent with previous reports showing high co-circulation of ORV in hospitalised patients during the influenza season, and confirmed that the viruses detected caused very similar ILI respiratory manifestations. ${ }^{19-21}$ Moreover, our results reflected the profile of respiratory viruses in children, which was the predominant group included in the study. Interestingly, although circulation of influenza and ORV coincided over time, the frequency of co-detection of influenza with ORV was very low, whereas co-detection of more than one ORV in a single patient was rather common along with RSV. This observation was consistent with those in our previous reports, and we hypothesise that low co-detection of influenza with other ORVs in these hospitalised patients was likely due to the higher pathogenicity of influenza. ${ }^{202}$

We then compared clinical and epidemiological data from patients with influenza infection and patients with ORV infection to evaluate the impact of these infections. Influenza was significantly more frequent in older patients and those with severe diseases, whereas comorbidities were associated with ORV infections. These findings reflected a lower adhesion to immunisation among older children, with the presence of severe disease a possible consequence of the higher virulence of influenza viruses, although no difference in mortality was observed between both groups. This contrasted with results from a previous study performed in Fortaleza, showing significant association between RSV infections and infant mortality. ${ }^{23}$ We believe that such a discrepancy might be due to the low number of positive cases observed in the present study.

Studies of influenza seasonality in Brazil show that viral circulation in the north-east region is more prevalent in the first 4 months of the year, coinciding with a period of higher humidity in that area, ${ }^{144}$ whereas viral circulation in the south and south-east appears associated with lower temperature and unlikely to be linked to humidity data. ${ }^{25} 26$ Our data confirmed this observation and reinforced the growing consensus that vaccinations in northeast Brazil are being carried out in a postseasonal period, with consequent low effectiveness.

The factors associated with the diversity of seasonal patterns displayed during influenza epidemics in the tropics are not completely understood. A previous study identified a seasonal southward-travelling wave across Brazil (from equatorial to temperate regions), suggesting that environmental issues play more important roles than population factors, such as immune function and levels of vitamin $\mathrm{D}$, in driving influenza epidemics in the country. ${ }^{6}$

Understanding the dispersion dynamics of influenza viruses in tropical regions is considered of crucial importance, given that several reports suggested that in these regions, influenza activity is more temporally diffuse and might contain foci for the emergence of new variants. ${ }^{6727}$ In addition to the need for a different influenza-immunisation schedule in Brazil, the composition of this vaccine should also be evaluated, because the antigenic composition for this region might differ from that recommended for the temperate region. ${ }^{7}$ However, information on strains circulating in tropical regions remains scarce, because most countries in these regions have low income, and health surveillance is not completely employed. Additionally, policies and resources need to be established to overcome the logistical challenges to implementing national immunisation programmes using different calendars and distinct antigenic compositions in Brazil. $^{7}$

This study contained some limitations. Patient recruitment during the seasonal period of influenza previously defined for two sites (Curitiba and Rio de Janeiro) might have contributed to low FLUB detection, although the frequency observed here was similar to that reported by the national surveillance programme. Additionally, the study was performed using data collected over the course of 1 year, which limited predictions of the impact of influenza longitudinally in these locales. There was a predominance of paediatric patients in the study population; therefore, data concerning ORV reflect mainly the profile of respiratory viruses in children. The low number of patients from Rio de Janeiro did not allow for an assessment of the impact and seasonality of viral respiratory infections in that region. Finally, the monitoring of weather conditions was performed by organisations linked to the states of each region of the country, therefore limiting the description of data to that from similar information made available by these institutions.

Despite these limitations, our results indicated that the model of active surveillance applied in our study represents a valuable tool for investigating the impact of respiratory viruses in hospitalised patients in distinct regions of Brazil. We also reinforced the importance of monitoring influenza infections to enable more adequate preventive measures for the population.

\section{Author affiliations}

${ }^{1}$ Departamento de Doenças Infecciosas, Universidade Federal do Paraná, Curitiba, Brazil

${ }^{2}$ Laboratório de Virologia, Universidade Federal do Paraná, Curitiba, Brazil ${ }^{3}$ Departamento de Patologia e Medicina Legal, Universidade Federal do Ceará, Fortaleza, Brazil

${ }^{4}$ Laboratório de Vírus Respiratórios e do Sarampo, Instituto Oswaldo Cruz, Fundacao Oswaldo Cruz, Rio de Janeiro, Brazil 
${ }^{5}$ Programa de Pós-graduação em Medicina Interna e Ciências da Saúde, Universidade Federal do Paraná, Curitiba, Brazil

${ }^{6}$ Hospital Quinta D'Or, Rio de Janeiro, Brazil

${ }^{7}$ Instituto Nacional de Infectologia, Fundação Oswaldo Cruz, Rio de Janeiro, Brazi

${ }^{8}$ Scientific Department, Fondation Merieux, Lyon, France

${ }^{9}$ Vaccines Research, FISABIO-Public Health, Valencia, Spain

Contributors FKP, VP and JP-B participated in the conception/design of the study, supervised the analysis and interpreted the data. MMS and SMR supervised the analysis, interpreted the data and wrote the preliminary manuscript. FEAM and IT supervised the data collection from Fortaleza and Rio de Janeiro, respectively. BCC performed the tests, assembled the data and wrote the preliminary manuscript. VMA, LAP, MBN and LRV performed the molecular tests and collected the data from Curitiba. All authors contributed to the writing and review of the manuscript and approved the final version.

Funding This work was supported by Sanofi Pasteur through the Global Influenza Hospital Surveillance Network and the Mérieux Foundation.

Competing interests None declared.

Patient consent Obtained.

Ethics approval Ethical Committee of each institution involved in the research: Instituto Nacional de Infectologia Evandro Chagas (INI/Fiocruz), Rio de Janeiro; Hospital de Clínicas-Universidade Federal do Paraná (HC/UFPR), Curitiba; and Hospital Infantil Albert Sabin (HIAS), Fortaleza.

Provenance and peer review Not commissioned; externally peer reviewed. Data sharing statement No additional data are available.

Open Access This is an Open Access article distributed in accordance with the Creative Commons Attribution Non Commercial (CC BY-NC 4.0) license, which permits others to distribute, remix, adapt, build upon this work non-commercially, and license their derivative works on different terms, provided the original work is properly cited and the use is non-commercial. See: http://creativecommons.org/ licenses/by-nc/4.0/

(c) Article author(s) (or their employer(s) unless otherwise stated in the text of the article) 2018. All rights reserved. No commercial use is permitted unless otherwise expressly granted.

\section{REFERENCES}

1. Bresee J, Hayden FG. Epidemic influenza--responding to the expected but unpredictable. N Engl J Med 2013;368:589-92.

2. World Health Organization. Influenza (seasonal) fact sheet 211. 2014 http://www.who.int/mediacentre/factsheets/fs211/en/ (accessed 13 Sep 2016).

3. Bloom-Feshbach K, Alonso WJ, Charu V, et al. Latitudinal variations in seasonal activity of influenza and respiratory syncytial virus (RSV): a global comparative review. PLoS One 2013;8:e54445.

4. Caini S, Huang QS, Ciblak MA, et al. Epidemiological and virological characteristics of influenza B: results of the Global Influenza B Study. Influenza Other Respir Viruses 2015;9:3-12.

5. Alonso WJ, Yu C, Viboud C, et al. A global map of hemispheric influenza vaccine recommendations based on local patterns of viral circulation. Sci Rep 2015;5:17214.

6. Alonso WJ, Viboud C, Simonsen L, et al. Seasonality of influenza in Brazil: a traveling wave from the Amazon to the subtropics. Am J Epidemiol 2007;165:1434-42.
7. de Mello WA, de Paiva TM, Ishida MA, et al. The dilemma of influenza vaccine recommendations when applied to the tropics: the Brazilian case examined under alternative scenarios. PLoS One 2009;4:e5095

8. Tamerius JD, Shaman J, Alonso WJ, et al. Environmental predictors of seasonal influenza epidemics across temperate and tropical climates. PLoS Pathog 2013;9:e1003194.

9. Castrucci MR. Factors affecting immune responses to the influenza vaccine. Hum Vaccin Immunother 2017:1-10.

10. da Cunha SS, Camacho LA, Santos AC, et al. Influenza vaccination in Brazil: rationale and caveats. Rev Saude Publica 2005;39:129-36.

11. Luna EJ, Gattás VL. Effectiveness of the Brazilian influenza vaccination policy, a systematic review. Rev Inst Med Trop Sao Paulo 2010;52:175-81.

12. Oliveira JF, Boing AF, Waldman EA, et al. Ecological study on mortality from influenza and pneumonia before and after influenza vaccination in the Northeast and South of Brazil. Cad Saude Publica 2013;29:2535-45.

13 Brazilian Health Ministry. Departamento de informática do SUS (DATASUS). http://sipni.datasus.gov.br/si-pni-web/faces/relatorio/ consolidado/coberturaVacinalCampanhalnfluenza.jsf (accessed 11 Dec 2016).

14. Moura FE, Perdigão AC, Siqueira MM. Seasonality of influenza in the tropics: a distinct pattern in Northeastern Brazil. Am J Trop Med Hyg 2009;81:180-3.

15. World Health Organization. CDC protocol of real-time RT-PCR for influenza A (H1N1). 2009 http://www.who.int/csr/resources/ publications/swineflu/realtimeptpcr/en/ (accessed 3 Oct 2015).

16. Biere B, Bauer B, Schweiger B. Differentiation of influenza B virus lineages Yamagata and Victoria by real-time PCR. J Clin Microbiol 2010;48:1425-7.

17. Barros EN, Cintra O, Rossetto E, et al. Patterns of influenza $B$ circulation in Brazil and its relevance to seasonal vaccine composition. Braz J Infect Dis 2016;20:81-90.

18. Bulletim BE. Informe técnico - Situação epidemiológica da influenza (CREMESP). http://www.cremesp.org.br/pdfs/Informe_tecnico_flu_ 17mar2016.pdf (accessed 11 Dec 2016)

19. Brittain-Long R, Nord S, Olofsson S, et al. Multiplex real-time PCR for detection of respiratory tract infections. J Clin Virol 2008;41:53-6.

20. Raboni SM, Stella V, Cruz CR, et al. Laboratory diagnosis, epidemiology, and clinical outcomes of pandemic influenza $A$ and community respiratory viral infections in southern Brazil. J Clin Microbiol 2011;49:1287-93.

21. Giamberardin HI, Homsani S, Bricks LF, et al. Clinical and epidemiological features of respiratory virus infections in preschool children over two consecutive influenza seasons in southern Brazil. J Med Virol 2016;88:1325-33.

22. Trombetta $\mathrm{H}$, Faggion $\mathrm{HZ}$, Leotte J, et al. Human coronavirus and severe acute respiratory infection in Southern Brazil. Pathog Glob Health 2016;110:113-8.

23. Alonso WJ, Laranjeira BJ, Pereira SA, et al. Comparative dynamics, morbidity and mortality burden of pediatric viral respiratory infections in an equatorial city. Pediatr Infect Dis $J$ 2012;31:e9-14.

24. Moura FE. Influenza in the tropics. Curr Opin Infect Dis 2010;23:415-20.

25. Tsuchiya LR, Costa LM, Raboni SM, et al. Viral respiratory infection in Curitiba, Southern Brazil. J Infect 2005;51:401-7.

26. Bellei N, Carraro E, Perosa A, et al. Patterns of influenza infections among different risk groups in Brazil. Braz $\mathrm{J}$ Infect Dis 2007;11:399-402.

27. Rambaut A, Pybus OG, Nelson MI, et al. The genomic and epidemiological dynamics of human influenza A virus. Nature 2008;453:615-9. 